\title{
Activity of selected aromatic amino acids in biological systems
}

\author{
Wirginia Krzyściak \\ Department of Medical Diagnostics, Jagiellonian University, Medical College, Pharmacy Faculty, Kraków, Poland
}

\begin{abstract}
Besides the structural function in proteins, aromatic amino acids are precursors of many important biological compounds essential for normal functioning of the human organism. Many of these compounds may be used as markers for identification of specific pathological states. Comprehensive knowledge about the metabolism of aromatic amino acids and mechanisms of action of their metabolites made it possible to develop effective treatments for many disorders. However, it should not be forgotten that in some pathological conditions, these compounds could not only be involved in the pathogenesis of many disease entities but could also be used as an important tool in prediction of many diseases. This paper contains a review of published literature on aromatic amino acids in the context of physiological processes of the human body and chosen social disorders, such as cancers; psychiatric disorders: depression, anxiety states, schizophrenia, bipolar affective disorders; neurodegenerative, and cardiovascular diseases; chronic kidney insufficiency or diabetes.
\end{abstract}

Keywords: tyrosine, kynurenic acid, reactive oxygen species

Received: 31 August, 2011; revised: 30 November, 2011; accepted: 09 December, 2011; available on-line: 15 December, 2011

\section{THE SIGNIFICANCE OF TYROSINE AND ITS METABOLITES IN PHYSIOLOGY AND PATHOLOGY}

Besides the structural function in proteins, aromatic amino acids are precursors of many important biological compounds, such as neurotransmitters and hormones, in the human organism. Catecholic amines, such as dopamine, norepinephrine, and epinephrine are synthesized from tyrosine (Tyr). In the first step, L-DOPA (L-3,4-dihydroxyphenylalanine) is formed as a result of tyrosine hydroxylase action (EC 1.14.16.2; HT). Then, L-DOPA undergoes fast decarboxylation to dopamine by aromatic L-amino acid decarboxylase (EC 4.1.1.28; LAAD) (Hadjiconstantinou \& Neff, 2008). This process occurs in neurons of substantia nigra, from which the neurotransmitter is transported to striatum and then released on nerve endings. In Parkinson's disease, neurons of the nigrostriatal pathway undergo degeneration that results in the impairment of dopaminergic conduction manifested by tremors at rest and bradykinesia (slowness of movements). After administration of L-DOPA to patients, the compound crosses the blood-brain border, elevates the concentration of dopamine in brain, and alleviates disease symptoms (Tedroff, 1999; Rowland, 2005). Oxidation stress and subsequent oxidation of proteins, lipids and nucleic acids also play a significant role in the damage of dopaminergic neurons. Dopamine is also produced in chromaffin cells of the adrenal me- dulla where it is later converted first to norepinephrine by dopamine $\beta$-hydroxylase (EC 1.14.17.1; DBH), then to epinephrine by phenylalanine-N-methyltransferase (EC 2.1.1.28; PNM'T). Blood flow-pressure is regulated by catecholamines generated in the adrenal medulla. They are released during stress situations. Increased release of catecholamines into the circulation was observed in neoplasms originating from phaeochromocytoma and in undifferentiated neuroblastic cells of the sympathetic system in ganglia and the adrenal medulla (neuroblastoma, ganglioneuroma). The increase in L-DOPA, norepinephrine and chromogranin A concentration in plasma was observed in phaeochromocytoma, which can be indicative of malignancy of this neoplasm. However, a decrease in norepinephrine and epinephrine levels in blood was observed in rare disorders of the adrenal medulla, such as atrophy of the medulla caused by congenital absence of adrenal cortex and dopamine $\beta$-hydroxylase deficiency (Fung et al., 2008).

Besides catecholamines, melanins (pigments providing protection against UV radiation) are also formed from tyrosine. In mammals, we distinguish two types of melanins: phenyl-melanin (red-brown colour) and eumelanin (dark-brown colour). Because of the presence of tyrosinase (EC 1.14.18.1), skin melanocytes have the ability to produce melanins. Tyrosinase catalyses the reaction of hydroxylation of tyrosine to L-DOPA and oxidation of L-DOPA to L-DOPA-quinone. This is a branch point of the pheno- and eumelanin synthesis pathway.

Melanoma is a result of the disorder of melanocyte function. In melanocytes with neoplastic changes, there is an increased synthesis of melanin. Melanins and their precursors occur in blood and in urine. The relationship between the L-DOPA/L-tyrosine ratio and the clinical condition in patients was established based on the studies on biomarkers for melanoma. This ratio significantly increases in patients with metastasis to local

e-mail address: wirginiakrzysciak@cm-uj.krakow.pl Abbreviations: AAA, aromatic amino acids; $A \beta$, amyloid- $\beta$; AIDS, acquired immune deficiency syndrome; 3-HAA, 3-hydroxyanthranilic acid; 3-HKA, 3-hydroxy-l-kynurenine; 5HT, 5-hydroxytryptamine; 5-HTTP, 5-hydroxytryptophan; CNS, central nervous system; $\mathrm{CRI}$, chronic renal insufficiency; CVD, chronic venous disease; CYP $1 \mathrm{~A} 1$, cytochrome P450 1A1; DBH, dopamine $\beta$-hydroxylase; ESRD, end-stage renal disease; HIOMT, hydroxyl-indol O-methyl transferase; HT, tyrosine hydroxylase; IDO, indoleamine 2,3-dioxygenase; INF- $\gamma$, interferon- $\gamma$; KYN, kynurenine; KYNA, kynurenic acid; LAAD, L-amino acid decarboxylase; L-DOPA, L-3,4-dihydroxyphenylalanine; LPS, lipopolysaccharides; NFTs, neurofibrillary tangles; PAH, phenylalanine hydroxylase; PIC, proinflammatory cytokines; PNMT, phenylalanine- $N$-methyltransferase; $Q A$, quinolinic acid; RNS, reactive nitrogen species; ROS, reactive oxygen species; $5-S-C D, 5-S-$ cysteinyldopa; SNAT, serotonin N-acetyltransferase; TDO, tryptophan 2,3-dioxygenase; TM, thrombomodulin; TNF-a, tumor necrotic factor; TRY, tryptophan; Tyr, tyrosine; vWF, von Willebrand Factor; $\mathrm{XA}$, xanthurenic acid; a7-nAchR, a7 nicotinic acetylcholine receptor. 




black and brown polymers

Figure 1. Tyrosine metabolites as components of pheomelanin and eumelanin pathways.

lymph nodes and therefore may be useful in early detection and effective treatment of the disease. In the case of patients without metastases, an elevated value of this ratio can indicate the worst prognosis. Other metabolites such as 5-S-cysteinyldopa (5-S-CD) of the pheomelanin pathway and, to a lower extent, 5-hydroxy-2-methoxy indole-2-carboxylic acid of the eumelanin pathway are used as markers of melanogenesis. However, the L-DOPA/L-tyrosine ratio seems to be a marker that is more sensitive and easier to evaluate (Figs. 1, 2) (Garnier et al., 2007).

In humans, tyrosine can be synthesized from phenylalanine in liver and kidneys with the participation of phenylalanine hydroxylase (EC 1.14.16.4; PAH). This process is significantly impaired in chronic renal insufficiency (CRI), which results in a decrease in the tyrosine/phenylalanine ratio in blood and tissues. In patients with CRI, oxidation stress occurs very often and results in the occurrence of oxidized tyrosine forms (chlorotyrosine and nitrotyrosine) in blood (Kopple, 2007).

\section{THE SIGNIFICANCE OF TRYPTOPHAN AND ITS METABOLITES IN PHYSIOLOGY AND PATHOLOGY}

Tryptophan is also a very important amino acid because two hormones, serotonin and melatonin, are synthesized based on it. The first step in the synthesis of serotonin is the synthesis of 5-hydroxytryptophan (5-HTP) due to tryptophan hydroxylase action (EC 1.14.16.4). Then, 5-HTP is converted into serotonin (5-hydroxytryptamine - 5HT) with the participation of LAAD (Mendelsohn et. al., 2009; Thomas et al., 2010). Serotonin is a neurotransmitter that is responsible for regulation of cognitive functions of the brain. Therefore, any serious disturbance of serotonergic conduction results in psychiatric disorders related to disturbances of the mood, such as: depression, anxiety, schizophrenia, bipolar affective disorders. In some patients suffering from depression, changes in the level of 5-HT serotonin, which plays a significant role in mood regulation, can be observed; however, the low level of 5-HT may also result from 


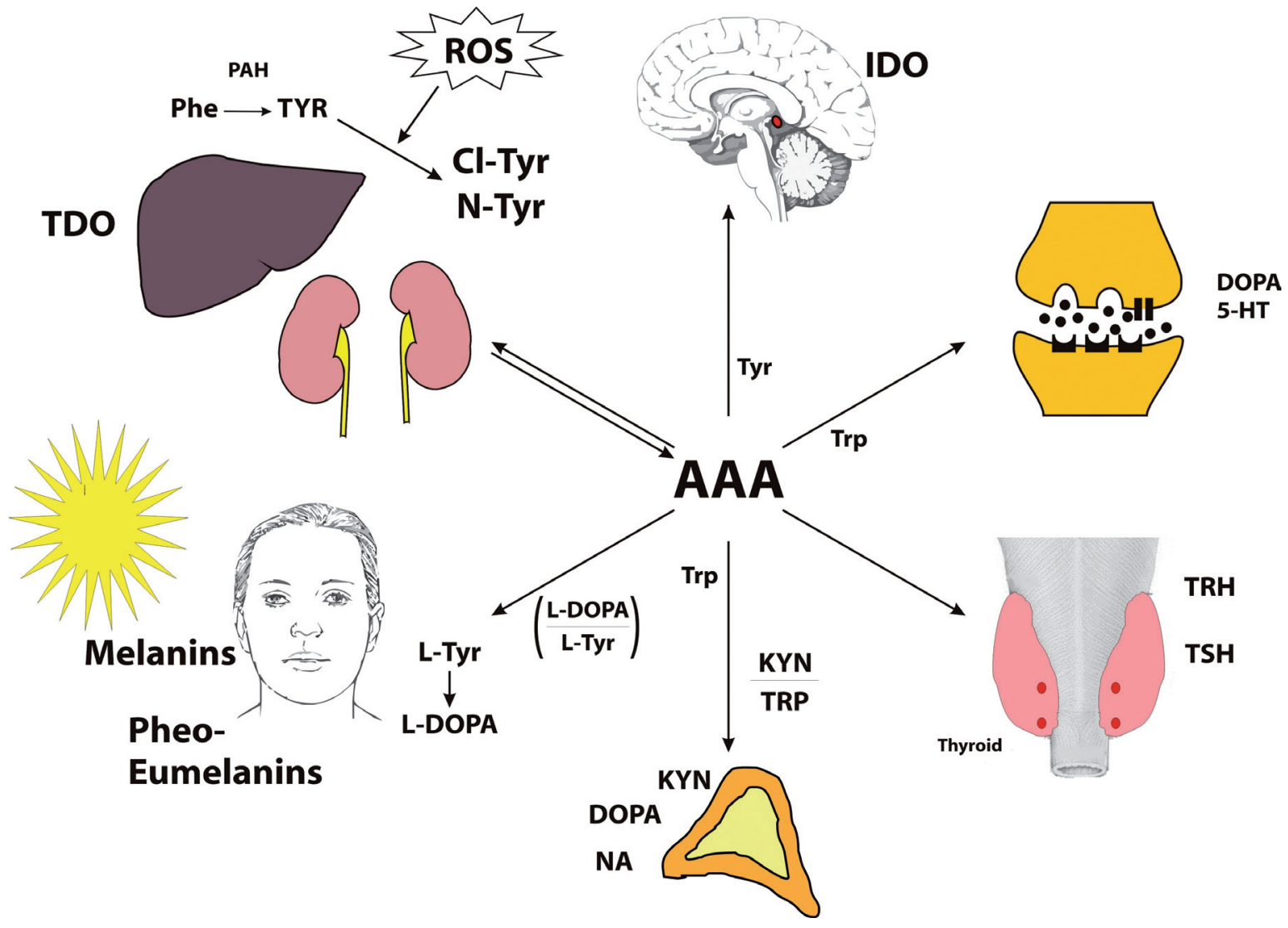

Figure 2. Activity of aromatic amino acids (AAA) in biological systems.

In liver and kidneys phenylalanine is converted to tyrosine with a contribution of phenylalanine hydroxylase (PAH). Tyrosine in turn is used in the synthesis of catecholamines (dopamine, epinephrine and norepinephrine) in the adrenal medulla and in the synthesis of pheomelanin and eumelanin in skin melanocytes, the first stage of which is L-DOPA formation. Elevated L-DOPA/L-Tyr index is observed in patients with malignant melanoma and metastases to local lymphoid nodules. Tyrosine is also an initial compound for the synthesis of thyroid gland hormones - thyroxine $\left(\mathrm{T}_{4}\right)$ and triiodothyronine $\left(\mathrm{T}_{3}\right)$. Other Tyr derivatives, like 3-Cl-D-Tyr or 3-N-D-Tyr, may be formed in conditions of oxidation, nitrification and chlorination stress. A neurotransmitter - serotonin (5-HT), which is also a melatonin precursor in the pineal gland, is synthesised from tryptophan. The main way of TRY transformation is the kynurenine pathway, whose main enzymes include indoleamine 2,3-dioxygenase (IDO) present in brain and numerous peripheral tissues, and tryptophan 2,3-dioxygenase (TDO) present in liver. Both these enzymes catalyze the same reaction of TRY conversion to kynurenine (KYN). IDO is an inducible enzyme subjected to activation in situations stressful for the organism, e.g. under the influence of proinflammatory cytokines. Elevated KYN/TRY index in patients with terminal renal insufficiency is a prognostic factor for the occurrence of heart and vascular complications.

lower concentration of tryptophan in the blood and its poor availability for the synthesis of this neurotransmitter in the brain (Merens et. al., 2008).

From the clinical point of view, it is recommended to quantify the amount of serotonin. Because its content in the saliva is strongly correlated with its level in the central nervous system (CNS), serotonin may be regarded in the future as a marker of neurodegenerative diseases. Interestingly, the content of serotonin is around two-times higher in the saliva of patients suffering from migraine than in the control population (Buczko et al., 2005).

In the pineal body, serotonin can be also used for the synthesis of another hormone, melatonin. Serotonin $\mathrm{N}$-acetyltransferase (EC 2.3.1.87; SNAT) converts serotonin to $\mathrm{N}$-acetylserotonin (NAS), from which melatonin is directly formed (N-actetyl-5-methoxytryptamine) with the participation of hydroxyl-indol O-methyl transferase (HIOMT). Secretion of melatonin is a subject to daily fluctuations. It is synthesized and released at night, therefore it is called a night hormone (Banach et al., 2011).

However, the serotonin pathway is only in small part responsible for tryptophan transformation. A significant majority, around $94 \%$ of tryptophan, is degraded to kynurenine (KYN). This reaction is catalyzed by two enzymes: indoleamine 2,3-dioxygenase (EC 1.13.11.52;
IDO) that occurs in kidneys, lungs, intestine, spleen, brain, epididymis, placenta, endocrine glands and in macrophages/monocytes, and tryptophan 2,3-dioxygenase (EC 1.13.11.11; TDO), which is present only in liver. In the further stages of tryptophan transformation, a series of compounds (kynurenines) with specific biological properties are formed. The IDO is an inducible enzyme that is sensitive to proinflammatory cytokines, contrary to TDO that is more active when higher concentrations of TRY are present in the blood.

There is information in the published literature, which indicates a relationship between elevated activity of IDO and the occurrence of cardiovascular diseases in patients with terminal kidney insufficiency (end-stage renal disease; ESRD). The impairment of endothelium function in patients with ESRD is directly related to the accumulation of kynurenines in blood plasma and elevated oxidation stress. Increase in the kynurenine/tryptophan ratio as a result of IDO activity, and the presence of oxidation stress markers can be a prognostic factor for the occurrence of a cardiovascular disease in patients with ESRD (Pawlak et al., 2009). It was also proved that an elevated level of metabolites of the kynurenic pathway $(\mathrm{KP})$ and the increase in oxidation stress are significantly correlated with the presence of markers of vascular en- 
dothelium dysfunction, thrombomodulin (TM) and von Willebrand factor (vWF) (Pawlak et al., 2009). Increase in kynurenines and, related to it, a decrease in the level of tryptophan is the early indicator and a linking factor of such diseases as hypertension, chronic kidney insufficiency, diabetes, and cardiovascular complications. Studies performed by Koening (2010) demonstrated that a significant increase of the KYN/TRY ratio occurs also in dialyzed patients and is correlated with the increase in the concentration of neopterin. This proves the relationship between enhanced degradation of tryptophan and inflammatory processes in patients (Koenig et al., 2010). Recent studies by Pawlak et al. (2010) linked the increase in the concentration of metabolites of the kynurenic pathway with an increased risk of the occurrence of Chronic Venous Disease (CVD) in patients with terminal insufficiency of kidneys who received peritoneal dialysis. The increase in the concentration of KYN, kynurenic acid (KYNA) and quinolinic acid (QA) was correlated with elevated levels of components of the haemostatic system, such as tissue factor and its inhibitor, tissue and urokinase plasminogen activator, the plasmin/ antiplasmin complex and others. Some metabolites of kynurenine can be used as a prognostic factor of the occurrence of cardiovascular complications in patients receiving peritoneal dialysis (Pawlak et al., 2010). Interestingly, the increase in IDO activity is induced mainly by interferon- $\gamma$ (INF- $\gamma$ ), which is released during the inflammatory process. However, activation of IDO can be induced by other proinflammatory cytokines as well, such as interferon- $\alpha(\mathrm{IFN}-\alpha)$ and tumor necrotic factor $(\mathrm{TNF}-\alpha)$. These compounds can enhance the INF- $\gamma$ inducibility of IDO. A condition of chronic inflammation with a constant release of proinflammatory cytokines (PIC) occurs in people in the advanced age. Therefore, a relationship between increased activity of the kynurenic pathway, neuroendocrine disorders and the occurrence of metabolic syndrome in people in the advanced age is studied (Oxenkrug, 2010).

Increased level of kynurenines in saliva was established in patients suffering from type 2 diabetes. However, this increased level is also related to the upregulation of IDO by proinflammatory cytokines, because visceral adipose tissue, regarded as the main cause of insulin resistance, releases many biologically active substances, including TNF- $\alpha$ and IFN- $\gamma$. (Brandacher et al., 2007). The effect of the derivatives of tryptophan on the development of diabetes can proceed in two ways. Firstly, QA inhibits synthesis of proinsulin in the pancreas as well as its release from $\beta$ cells. Moreover, xanthurenic acid (XA) forms a complex with insulin thereby insulin cannot bind to its receptor (Buczko et al., 2006). Studies on insulin resistance showed significantly elevated activity of another enzyme of the kynurenic pathway, ACMSD, in hepatocytes of rats with artificially induced diabetes (Sasaki et al., 2009). This is another element demonstrating a relationship between this particular disease entity and enhanced metabolism of tryptophan. Similarly to the interrelation between diabetes and changes in the oral cavity, the influence of tryptophan and its derivatives on the level of carbohydrates have a mutual character and needs more studies to be better known. The level of kynurenines increases rapidly in bacterial infections, which is related to stimulation of the immunological system, release of proinflammatory cytokines and direct activation of IDO by bacterial lipopolysaccharides (LPS) in the oral cavity. Therefore, the assumption that the metabolism of tryp- tophan is enhanced in inflammatory states of the gums and in parodontium diseases is totally justified (Buczko et al., 2005).

Quinolinic acid is also an agonist of the NMDA receptor (NMDAR), whose stimulation results in the influx of calcium ions into cells followed by activation of proteases and generation of reactive oxygen species (ROS) and reactive nitrogen species (RNS) such as superoxide radical anion $\left(\mathrm{O}_{2}{ }^{-}\right)$and nitrogen oxide $(\mathrm{NO})$. These processes can result in neuron damage as revealed in studies on the etiology of neurodegenerative diseases. As the agonist of the NMDA receptor, QA is an important neurotoxic agent. In contrast to QA, KYNA, which is an antagonist of the NMDA receptor demonstrates neuroprotective action. The physiological level of QA does not have a negative effect on neurons; however, a slight increase in its concentration (an order of a few micromoles) is enough to cause damage. In patients with acquired immunodeficiency syndrome (AIDS), who also suffered from dementia, there was a 20 -fold increase in QA concentration in cerebrospinal fluid (Stone et al., 2003). A similar mechanism of neuron damage was observed in patients with depression. As the study proved, lowering of 5 -HT level in brain is not the only cause of mood disorders in this syndrome. Compounds that were mentioned earlier in the paper, which are generated during the catabolism of tryptophan through the kynurenic pathway, also play a meaningful role. Increased activity of the hypothalamus - pituitary - adrenal glands axis together with increased glucocorticosteroids level accompany depression as does general inflammatory state coupled with activation of immunological cells and release of proinflammatory cytokines. These factors influence the induction of TDO (glucocorticosteroid) and IDO (PID). In women, the level of IDO activation is significantly higher than in men, which means that the risk of depression is significantly higher in the female gender. Excessive activity of IDO and TDO results in a decrease in TRY concentration in the periphery and in the brain, limiting its availability for the synthesis of 5-HT. Kynurenines, when generated in excessive amounts, mostly demonstrate neurotoxic and neurodegenerative action. One example is the action of quinolinic acid as an agonist of the NMDA receptor. Another compound with high neurotoxic potential is 3-hydroxy-l-kynurenine (3-HKA) and its metabolite, 3-hydroxyanthranilic acid (3-HAA). In this case, neuron damage is caused by the action of free radicals: $\mathrm{O}_{2}{ }^{-}$, hydroxyl radical $\left(\mathrm{OH}^{*}\right)$ and superoxide $\left(\mathrm{H}_{2} \mathrm{O}_{2}\right)$. Moreover, all three compounds impair mitochondrial functions and finally result in a decrease in the efficacy of the electron transport chain and ATP production. Both the dysfunction of mitochondria and oxidation stress play an important role in the pathophysiology of depression. QA, through its proinflammatory properties, has an effect on the activation of immunological cells of microglia and on the release of PID, which additionally enhance the activity of IDO. However, tryptophan and some of its degradation products, such as kynurenine, kynurenic acid and xanthurenic acid, due to anti-inflammatory properties, induce a negative feedback on cells of the immunological system, limiting production of proinflammatory cytokines and thereby the activity of IDO (Fig. 3).

Figure 3 shows that the (neuro)inflammation in depression, which is characterized by an increased production of interleukin-1 $\beta$ (IL-1) $\beta$, IL-6, tumor necrosis factor- $\alpha(\mathrm{TNF} \alpha)$ and interferon (IFN) $\gamma$, induces indoleamine 2,3-dioxygenase (IDO) in the blood and in 


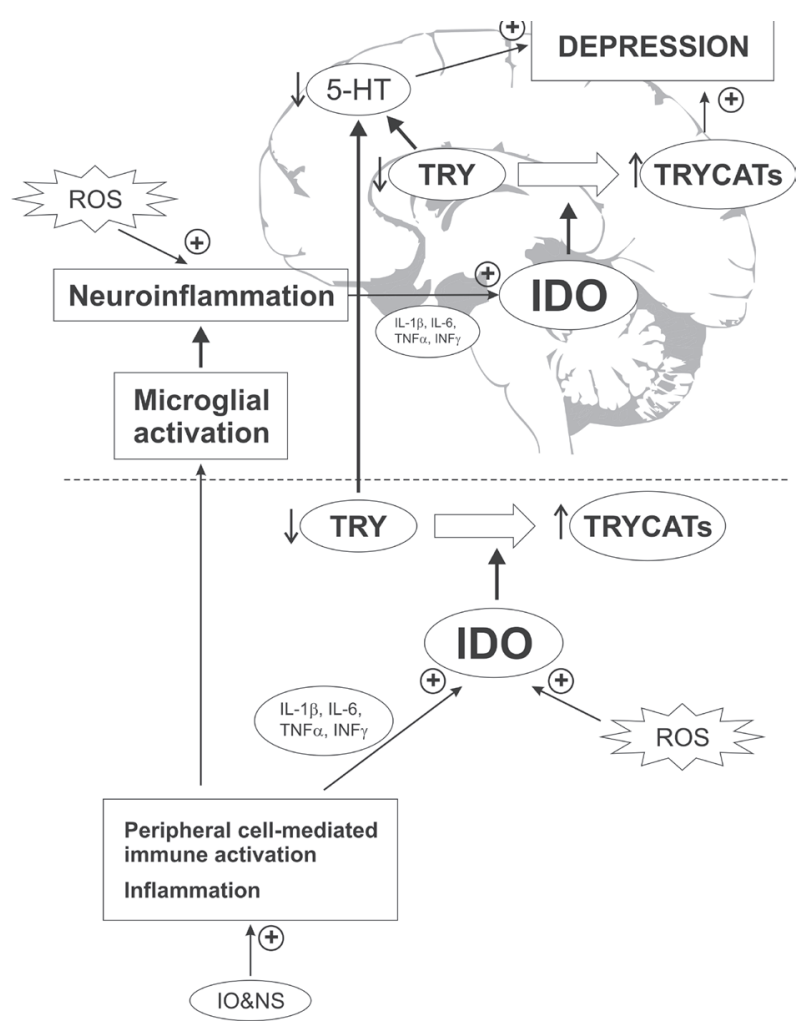

Figure 3. The TRYCAT pathway and indoleamine 2,3-dioxygenase (IDO) and their interconnections with peripheral and central immune, inflammatory, oxidation and nitrosative stress (IO\&NS) pathways.

the brain. This results in reduced tryptophan (TRY) levels and an increased production of tryptophan catabolites (TRYCATs). It may be hypothesized that decreased tryptophan and increased TRYCAT levels may attenuate the inflammation-induced depressive behaviors.

There are assumptions that depression may be used as an early prognostic factor of Alzheimer's disease. Both depression and dementia related to Alzheimer's disease have a common background, which is the inflammatory state and activation of the kynurenic pathway (Maes et al., 2011). In Alzheimer's disease, extracellular accumulation of amyloid- $\beta$ (A $\beta$ ) in the form of plaques, excessive phosphorylation of tau protein and formation of neurofibrillary tangles (NFTs) was established. It is argued that A $\beta$ activates Hortega cells and astrocytes by inducing inflammatory process and increased synthesis of toxic kynurenines. The action of 3-HKA and 3-HAA, through the release of ROS, can result in cell damage. Besides excitotoxicity induced by activation of the NMDA receptor and generation of oxidation stress, QA also has the ability to induce apoptosis in astrocytes and to timulate hyperphosphorylation of the tau protein, probably through lowering the activity of phosphatases. All these factors result in progressive neurodegeneration and dementia characteristic for Alzheimer's disease (Bonda et al., 2010). In addition, a relationship between the metabolites of tryptophan degradation and the occurrence of schizophrenia was also established. Kynurenic acid is an antagonist of the $\alpha 7$ nicotinic acetylcholine $(\alpha 7-n A c h R)$ and NMDA receptors. KYNA has neuroprotective action. Through inhibition of the NMDA receptor, KYNA limits the phenomenon of cellular excitotoxicity mediated by this receptor. It also protects brain from hypoxia. Therefore, improper KYNA concentration can result in disturbances in cholinergic and glutaminergic transmission and thereby contribute to the onset of diseases with a neurological and psychiatric background. Inhibition of $\alpha 7$-nAchR through KYNA results in reduction of the glutamate level and a decrease in the extracellular concentration of dopamine. Lowering of the KYNA level results in an increase in dopamine concentration in striatum (Rossi et al., 2008).

A hugely interesting and important issue is the participation of some tryptophan metabolites in carcinogenesis. Indolic derivatives of tryptophan, tryptamine (TA) and indole-3-acetic acid (IAA), are known as endogenous ligands which activate aryl hydrocarbon receptor; AhR. The transformed ligand - receptor complex is transported to the nucleus and, by binding to a specific DNA sequence it acts as a transcription factor enhancing expression of some genes including the gene encoding cytochrome P450 1A1 (CYP 1A1). CYP 1A1, together with other enzymes of the I and II stage of biotransformation, can transform exogenous procancerogens into compounds that are able to directly damage DNA. In addition, tryptophan metabolites that are not carcinogens by themselves can promote a neoplastic process within the urinary bladder in the presence of carcinogenic substances such as 2-naphthylamine and 4-aminobiphenyl. Increase in metabolites of the kynurenic pathway, such as $\mathrm{KN}, 3-\mathrm{OHAA}, \mathrm{XA}$ and others was observed in urine in patients with urinary bladder cancer. This observation confirmed the accumulation of the kynurenic pathway metabolites in the urinary bladder. Synthesis of xanthurenic acid increases in states of vitamin $\mathrm{B}_{6}$ deficiency. A diet rich in tryptophan coupled with $\mathrm{B}_{6}$ deficiency increases the risk of urinary bladder cancer. Tryptophan can be also metabolized to indole which is secreted to urine and acts as a carcinogenic agent (Chung \& Gadupudi, 2011). Moreover, a number of substances of a mutagenic, genotoxic and carcinogenic character, such as phenolic and indolic compounds, can be formed from tryptophan and other aromatic amino acids remaining in the large intestine after bacterial degradation. Contribution of these compounds to the pathogenesis of large intestine cancer was proved (Nowak \& Libudzisz, 2008). Studies performed by Suzuki et al., demonstrated an increase in IDO activity in patients with lung cancer together with higher kynurenine concentration and lower tryptophan concentration in their sera. Elevated concentration of KYN probably has an effect on the development of immunological tolerance towards neoplastic cells through proliferation inhibition and induction of apoptosis of lymphocytes T. Additionally, it was established that increase in IDO activity is correlated with progression of the disease. However, there is no answer to the question how activation of IDO promotes lung cancer. The most probable scenario is participation of INF- $\gamma$ that is endogenously released within cancer cells (Suzuki et al., 2010). Similar situation occurs in prostate cancer. Increased activity of IDO was detected in lymphatic vessels of cancer patients and in neoplastic tissue in mice with artificially induced prostate cancer. The KYN/TRY ratio was significantly elevated in animals with detectable cancer. Increase in IDO activity, like in the above example, was related with tumor-induced immunosupression. Lowering of IDO activity resulted in a delay in tumor growth (Källberg et al., 2010).

Another problem, which is gaining more and more attention recently, is oxidation stress. It was proved that tryptophan derivatives are among the strongest antioxidants, but they do not react with all free radicals. 
The 3-hydroxykynurenine, 3-hydroxyanthranilic acid and other kynurenines have the ability to scavenge $\mathrm{O}_{2}{ }^{-}-$ (Brandacher et al., 2007). Activation of IDO during immunological reaction contributes to the control of the oxidation stress. However, $\mathrm{NO}$ and $\mathrm{H}_{2} \mathrm{O}_{2}$ inhibit IDO activity (Thomas \& Stocker, 1999). On the other hand, as already mentioned in the paper, 3-HKA and 3-OHAA can generate free radicals in some conditions and thereby evoke neuron damage during the course of neurodegenerative diseases.

As it was presented in this paper, aromatic amino acids and their derivatives exert many important functions in the organism under normal physiological conditions. Moreover, many of these compounds may be used as markers for identification of specific pathological states. Better knowledge of the metabolism of aromatic amino acids and the mechanisms of action of their metabolites will contribute to the development of effective treatments for many disorders. However, it should not be forgotten that in some pathological conditions, these compounds could be also involved in the pathogenesis of many disease entities.

\section{REFERENCES}

Banach M, Gurdziel E, Jędrych M, Borowicz KK (2011) Melatonin in experimental seizures and epilepsy. Pharmacol Rep 63: 1-11.

Bonda DJ, Mailankot M, Stone JG, Garrett MR, Staniszewska M, Castellani RJ, Siedlak SL, Zhu X, Lee H, Nagaraj RH, Smith MA (2010) Indoleamine 2,3-dioxygenase and 3OH-kynurenine modifications are found in the neuropathology of Alzheimer disease. Redox Rep 15: 161-168.

Brandacher G, Hoeller E, Fuchs D, Weiss HG (2007) Chronic immune activation underlies morbid obesity: Is IDO a key player? Current Drug Metabolism 8: 289-295.

Buczko P, Cylwik D, Stokowska W (2005) Metabolism of tryptophan via kinurenin patchway in the saliva. Postepy Hig Med Dosw 59: 283289 (in Polish).

Buczko P, Stokowska W, Górska M, Kucharewicz I, Pawlak D, Buczko W (2006) Tryptophan Metabolites via kynurenine pathway in saliva of diabetic patients. Dent Med Probl 43: 21-25.

Chung KT, Gadupudi GS (2011) Possible roles of excess tryptophan metabolites in cancer. Environ Mol Mutagen 52: 81-104.

Fung MM, Viveros OH, O'Connor DT (2008) Diseases of the adrenal medulla. Acta Physiologica 192: 325-335.

Garnier JP, Letellier S, Cassinat B, Lebbé C, Kerob D, Baccard M, Morel P, Basset-Seguin N, Dubertret L, Bousquet B, Stoitchkov K, Le Bricon T (2007) Eur J Cancer 43: 816-21.

Hadjiconstantinou M, Neff NH (2008) Enhancing aromatic L-amino acid decarboxylase activity: implications for L-DOPA treatment in Parkinson's disease. CNS Neurosci Ther 14: 340-51.

Källberg E, Wikström P, Bergh A, Ivars F, Leanderson T (2010) Indoleamine 2,3-Dioxygenase (IDO) Activity influence tumor growth in the TRAMP prostate cancer model. The Prostate 70: 1461-1470.
Koenig P, Nagl C, Neurauter G, Schenech H, Brandacher G, Fuchs D (2010) Enhanced degradation of tryptophan in patients on hemodialysis. Clin Nephrol 74: 465-70.

Kopple JD (2007) Phenylalanine and tyrosine metabolism in chronic kidney failure. The I Nutr 137: 1586-1590.

Maes M, Leonard BE, Myint AM, Kubera M, Verkerk R (2011) The new '5-HT' hypothesis of depression: Cell-mediated immune activaction induces indoleamine 2,3-dioxygenaze, which leads to lower plasma tryptophan and an increased synthesis of detrimental tryptophan catabolites (TRYCATs), both of which contribute to the onset of depression. Prog Neuropsychopharmacol Biol Psychiatry 35: 702-21.

Mendelsohn D, Riedel WJ, Sambeth A (2009) Effects of acute tryptophan depletion on memory, attention and executive functions: A systematic review. Neur Biobehav Rev 33: 926-952.

Merens W, Booij L, Haffmans PJ, Does AJ (2008) The effects of experimentally lowered serotonin function on emotional information processing and memory in remitted depressed patients. $J$ Psychopharmacol 22: 653-662.

Nowak A, Libudzisz Z (2008) Carcinogenic activity of intestinal microorganisms. Nauka. Technologia. Jakość 6: 25-39 (in Polish).

Oxenkrug GF (2010) Metabolic syndrome, age-associated neuroendocrine disorders, and dysregulation of tryptophan-kynurenine metabolism. Ann N Y Acad Sci USA 11: 1-14.

Pawlak K, Domaniewski T, Myśliwiec M, Pawlak D (2009) The kynurenines are associated with oxidation stress, inflammation and the prevalence of cardiovascular disease in patients with end-stage renal disease. Atherosclerosis 204: 309-314.

Pawlak K, Domaniewski T, Myśliwiec M, Pawlak D (2009) Kynurenines and oxidative status are independently associated with thrombomodulin and von Willebrand factor levels in patient with endstage renal disease. Thrombosis Res 124: 452-457.

Pawlak K, Myśliwiec M, Pawlak D (2010) Haemostatic system, biochemical profiles, kynurenines and the prevalence of cardiovascular disease in peritoneally dialized patients. Thrombosis Res 125: 40-45.

Rossi F, Garavaglia S, Montalbano V, Walsh MA, Rizzi M (2008) Crystal structure of human kynurenine aminotransferase ii, a drug target for treatment of schizofrenia. J Biol Chem 283: 3559-3566.

Rowland LP (2005), Primary lateral sclerosis, hereditary spastic paraplegia, and mutations in the alsin gene: historical background for the first International Conference. Amyotroph Lateral Scler Other Motor Neuron Disord 6: 67-76.

Sasaki N, Egashira Y, Sanada H (2009) Production of L-tryptophan-derived catabolites in hepatocytes from streptozocin-induced diabetic rats. Eur J Nutr 48: 145-153.

Stone TW, Mackay GM, Forrest CM, Clark CJ, Darlington LG (2003) Tryptophan metabolites and brain disorders. Clin Chem Lab Med 41: 852-859.

Suzuki Y, Suda T, Furuhashi K, Suzuki M, Fujie M, Hahimoto D, Nakamura Y, Inui N, Nakamura H, Chida K (2010) Increased serum kynurenine/tryptophan ratio correlates with disease progression in lung cancer. Lung Cancer 67: 361-365.

Tedroff JM (1999) Functional consequences of dopaminergic degeneration in Parkinson's disease. Adv Neurol 80: 67-70.

Thomas DM, Angoa Pérez M, Francescutti-Verbeem DM, Shah MM, Kuhn DM (2010) The role of endogenous serotonin in methamphetamine-induced neurotoxicity to dopamine nerve endings of the striatum. J Neurochem 115: 595-605.

Thomas SR, Stocker R (1999) Redox reactions related to indoleamine 2,3-dioxygenase and tryptophan metabolism along the kynurenine pathway. Redox Rep 4: 199-220. 